\title{
Reframing Fetal Alcohol Spectrum Disorder: Studying Culture to Identify Communication Challenges and Opportunities
}

\author{
Nathaniel Kendall-Taylor ${ }^{1}$, Marissa Fond ${ }^{1}$ \\ ${ }^{1}$ The Frame Works Institute, USA \\ Correspondence: Nathaniel Kendall-Taylor, The Frame Works Institute, USA. \\ Received: October 6, 2017 \\ Accepted: October 31, 2017 \\ Online Published: November 1, 2017 \\ doi:10.11114/smc.v5i2.2687 \\ URL: https://doi.org/10.11114/smc.v5i2.2687
}

\begin{abstract}
Implicit cultural understandings challenge those working to increase public awareness and support for programs to prevent and address fetal alcohol spectrum disorder (FASD). Understanding these cultural beliefs reveals key challenges that communicators face; it also helps identify opportunities to foster public engagement and build support for policies and programs that are important for reducing the prevalence of FASD as a public health issue. Through a series of interviews with members of the public in Manitoba, Canada, we identify the cultural models that members of the Manitoban public draw on to make sense of this issue. These models and their implications are used to create a set of recommendations that can improve understanding of the issue, increase issue salience, and generate support for solutions. While the research presented is specific to Manitoba, findings have significance for those working on FASD in other areas and for those working on other public health and science translation projects.
\end{abstract}

Keywords: culture and health, qualitative research, fetal alcohol spectrum disorder, science translation, cultural models

\section{Introduction}

Fetal alcohol spectrum disorder (FASD) is a complex social issue and an increasingly important public health problem. Evolving diagnostic criteria and high rates of comorbidity make its prevalence difficult to determine (Burd, 2016; Chudley et al., 2005), but rates are currently estimated at 22 per 1,000 live births globally, and rates in the United States and Canada at 34 and 31 per 1,000 live births, respectively (Millichap \& Millichap, 2014; Riley, 2011; Roozen et al., 2016). Increased prevalence entails increased costs. Although comprehensive assessments of the full economic costs of FASD are scarce, the combined public health costs have been recently estimated to be $\$ 5.4$ billion in the United States and $\$ 5.3$ billion in Canada (Popova et al., 2011; Stade et al., 2009).

Despite a growing recognition of FASD as an emerging public health issue among researchers, clinicians, and community advocates, we find that the general public does not understand FASD in the ways that experts do. Many of these public understandings threaten support for programs and policies that can prevent and address FASD.

Research on public understanding of FASD is limited, with only a small number of studies examining public discourse on this issue. These studies have analyzed popular news media in the United Kingdom, United States, and Australia, and have identified a set of common themes (Bell, McNaughton, \& Salmon, 2009; Lowe, Lee, \& Yardley, 2010; Connolly-Ahern \& Broadway, 2008). Among them, first, women are consistently portrayed as stewards of, and responsible for, their children's wellbeing. For example, pregnant women are often depicted as vessels, responsible for providing a safe "home" for unborn children (Benoit, Magnus, Phillips, Marcellus, \& Charbonneau, 2015; Bell, McNaughton, \& Salmon, 2009; Rabuzzi, 1994). FASD is most often discussed as a product of a woman's personal decisions with little to no attention paid to social or environmental factors. It has been argued that this contributes to the idea of the bad or dangerous woman who is deserving of shame (Eguiagaray, Scholz, \& Giorgi, 2016; Connolly-Ahern \& Broadway, 2008). Media reports on FASD have also been found to highlight the victimization of children, the idea of a "damaged child" presented in sympathetic tones, and compassion for both the child and caregiver (Lowe, Lee, \& Yardley, 2010; Connolly-Ahern \& Broadway, 2008).

Some research has found that media attention to FASD shifted thematically following the 1988 requirement of alcohol warning labels in the United States (Golden, 2000; Lowe et al., 2010). Prior to 1988, coverage had focused on white middle-class women and the associated public health concerns of FASD broadly. Post-1988, pregnant women and mothers who drank were increasingly depicted as people of color and as a danger to society (Golden, 2000). Coverage 
also shifted to reflect the growing public conception that there is "no safe amount" and total abstention from alcohol when pregnant is ideal (Lowe et al., 2010).

Another line of research focuses on the roles of public health professionals and service providers in influencing women's behavior (Benoit et al., 2015; Pei, Leung, Jampolsky, \& Alsbury, 2016). Studies have analyzed content in existing public health campaigns with a focus on professionals' conceptualizations of problematic alcohol use among clients (Benoit et al., 2015; Ryan \& Bonnett, 2006). Others have examined communications between service providers and clients, focusing on interpersonal service delivery methods when working with people who have FASD, pregnant women, mothers, and women who drink alcohol (Stewart \& Glowatski, 2014).

To add to existing literature, the research presented here focuses on a different aspect of the issue. Instead of examining media representations, practitioner understandings, or patient-provider interactions, we concentrate on increasing our understanding of the ways that members of the public make sense of FASD and process information related to this public health issue. We do this through a set of in-depth, cognitive interviews conducted in Manitoba, Canada. We find a set of cultural models (D'Andrade, 1995; Quinn \& Holland, 1987) —implicit but widely shared beliefs_about motherhood, substance use, individual choice, responsibility, and morality that influence how people process information about FASD. Some of these implicit understandings and assumptions act as barriers to increased public support for policies and practices that seek to prevent and address this issue.

This does not ignore the fact that people also have different ways of understanding complex issues like FASD - differences related to personal experiences and sub-group culture and identities. However, there are also common patterns of thinking that underlie diverse individual experiences and sub-group identities (D'Andrade, 2005; D'Andrade, 1987; Shore, 1996). Analyzing these shared patterns of thinking can be used to develop public health communication strategies to help shift the public discourse about FASD in Manitoba, ultimately generating greater support for programs and policies that have been shown to be effective.

We find that members of the Manitoban public have ways of thinking about FASD that impede their ability to support public health solutions on this issue. We find that the lack of support for FASD-related programs and policies is driven not by a lack of caring, but rather by culturally motivated understandings of the issue that make it hard to see public policy as a viable solution. A better understanding of culture in mind (Shore, 1996) and how it shapes thinking is a tool for creating more effective communications that promote better policies and solutions. Without an analysis of the culturally influenced patterns that structure thinking about social issues, advocates and experts are ill-equipped to communicate effectively, and may craft messages in ways that create backfire effects and that work against their goals of raising issue salience and generating policy support. With a map of the cultural models that shape public thinking, communicators change the information ecology of an issue-making certain policies less politically costly and inaction risky for decision-makers (Benford \& Snow, 2000) - to increase understanding of FASD in ways that help drive policy initiatives.

We focus on the following research question:

Research question: What are the cultural understandings that shape public perceptions of FASD in Manitoba, and how are these understandings applied to make sense of information on this issue?

In addition to this research question, we attempt to answer the following more interpretative question: How can communicators use this understanding of culture in mind to create more effective messages?

\section{Research Method}

To elicit evidence of cultural models, we conducted 20 in-depth interviews with Manitobans in the months of September and October of 2016 (Fond, Kendall-Taylor, Volmert, Gertein Pineau, \& L'Hôte, 2017). Half of the interviews were conducted in person in Winnipeg, while half were conducted via videoconference; this was done to draw participants from across the province, including more remote rural areas.

A professional marketing firm recruited participants, and they were selected to represent variation that loosely represented provincial census figures. Specific demographic variables of interest included ethnicity, gender, age, residential location (i.e., urban, suburban, rural), educational background (as a proxy for socioeconomic status), political views (as self-reported during the screening process), religious involvement, and family situation (married, single, with children, without children, age of children). The sample was composed as follows: 
Table 1. Interview participants

\begin{tabular}{l|l|l}
\hline Demographic category & & Sample \\
\hline Gender & Women & 11 \\
& Men & 9 \\
\hline Race/ethnicity & White & 13 \\
& Indigenous/Métis & 5 \\
& Black & 1 \\
& Hispanic & 1 \\
\hline Political views & Progressive Conservative & 9 \\
& Liberal & 8 \\
& Green Party & 3 \\
\hline Age & Mean & 40 \\
& Range & $20-62$ \\
\hline Education & High school degree & 5 \\
& Some college & 5 \\
& College degree & 6 \\
& Graduate degree & 4 \\
\hline Marital status & Married & 8 \\
& Not married & 12 \\
\hline Parental status & Is a parent & 9 \\
& Is not a parent & 11 \\
\hline
\end{tabular}

Researchers use cultural models interviews-one-on-one, semi-structured interviews lasting approximately two hours - to capture the broad sets of assumptions that participants use to make sense of a concept or topic area (Kendall-Taylor, 2012; Quinn, 2005a; D'Andrade, 2005), in this case, issues related to FASD. Interviews began with elicitations of participants' thinking about pregnancy, followed by substance use, and then FASD. Questions covered issues of FASD prevalence, causes, and effects; issue responsibility; and solutions. The goal of the interviews was to examine the cultural models that participants use to make sense of these issues, so researchers gave participants latitude to follow topics in directions that participants deemed relevant. All interviews were recorded and transcribed with participants' written and verbal consent.

Elements of grounded theory (Strauss \& Corbin, 2015; Strauss, 2005) and cultural models analysis (Quinn, 2005b) were applied to identify cultural models. First, three researchers independently identified common ways of talking across the sample to reveal assumptions, relationships, logical steps, and connections that were commonly made but taken for granted throughout an individual's interview and across the set of interviews. Analysis also revealed conflicting models that people brought to bear on the same issue. In such cases, one of the conflicting ways of understanding was typically found to be dominant over the other, in the sense that it more consistently and deeply shaped participants' thinking. Second, the three researchers shared their findings and re-analyzed the data to remove any instances of themes that had not surfaced across all three independent analyses. This validation suggests that the cultural models identified are indeed shared and strong in people's thinking.

That said, while the study's sample size and the length of each interview allow for the analysis of people's deep assumptions, the analysis does not lend itself to quantification. We do not provide relative frequencies of each cultural model described in the Findings below because the semi-structured nature of the interviews and the unpredictable emergence and shifts of topics mean that comparing tokens or codes across interviews is potentially misleading.

Excerpts from interviews are provided below to characterize themes and assumptions that emerged from analysis. Findings are thus representative of the data set but are represented by a small number of quotes. Presenting findings through "exemplary quotes" is standard practice in thematic analysis (Kempton, 1987; Strauss \& Corbin, 2015; Strauss, 2005).

In addition to describing patterned implicit understandings that run through participant interview data, we extend the analysis to draw implications of these findings for those communicating about FASD. These are interpretive points intended to generate novel hypotheses that could be tested in future research as well as to provide communicators with initial recommendations for how to use these findings to create more effective messages about FASD.

\section{Findings}

We find a set of clear and consistent patterns in the public's thinking about FASD.

First, participants were quite familiar with FASD, though they often referred to it as "FAS," or simply "fetal alcohol." 
Though participants were not told that their interview would be about FASD, when the conversation turned to the topic of pregnancy, all participants brought up alcohol consumption without the interviewer introducing the issue, and more than half of participants mentioned FASD specifically. This suggests that these issues were top of mind for participants and that there is a general awareness of FASD.

All participants asserted that women should not drink while pregnant and that alcohol, tobacco, and drug use during pregnancy were salient concerns.

Researcher: I want to start by talking about pregnancy, generally speaking. When you think about pregnancy, what comes to mind? ${ }^{1}$

Participant: Two things that come to my mind would be: don't have any alcohol or don't smoke when you're pregnant.

The clear and unequivocal view that pregnant women should not drink alcohol is based on a set of cultural models. These cultural models strongly influence attitudes and opinions about FASD, specifically in how participants think about what causes FASD and what can be done to address this issue.

\subsection{Foundational Cultural Models of Pregnancy and Responsibility}

The four cultural models described below were powerful influencers of people's thinking about what pregnancy means and what responsibilities it involves.

\subsubsection{Individualism}

The core of this cultural model, which has been found to shape public thinking about other social issues in North American culture (Lindland, Kendall-Taylor, Haydon, \& Fond, 2016; Kendall-Taylor, McCollum, \& Manuel, 2009; Hirschman, 2003; Triandis, 1989), is the assumption that the outcomes that people experience-specifically outcomes related to health and wellbeing - are primarily, if not exclusively, the result of individual choices, drive, and willpower. According to this assumption, contextual factors are largely irrelevant because outcomes are the result of individuals making good choices (or not). When participants were thinking about FASD, Individualism led them to a focus on a woman's choices, most typically, the choice to follow a healthy diet, exercise frequently, and get sufficient rest. In participants' thinking, these are the primary determinants of a successful pregnancy.

Drink plenty of healthy fluids, keep exercising, eat healthy foods while you're pregnant, and get plenty of sleep as well.

A woman should take care of herself. She should take prenatal vitamins. She should seek medical care. She should be thinking of the best interest of her child.

She needs to think about the health of their baby. And she needs to keep that mindfulness of healthy diet and proper nutrition and being aware of chemicals that she's around. Not putting herself in situations where the air could be compromised.

Individualism, and the primacy of personal choice and willpower in determining wellbeing, led participants to see unhealthy choices as the result of poor decision-making and to hold the individual in question at fault.

\subsubsection{The Gatekeeper}

In a slightly more specific version of Individualism, participants understood a woman who is pregnant as the gatekeeper to the fetus - controlling the substances to which the fetus is exposed through the decisions that she makes to consume, or not consume, certain substances. The components - or constituent propositions - of this model are as follows: (1) the pregnant woman decides what enters her body, (2) what enters the pregnant woman's body enters the fetus, and (3) substances that enter the fetus affect and determine its development and wellbeing. In participants' thinking, a woman is solely responsible, via her gatekeeping function during pregnancy, for fetal development and wellbeing.

Science has made it so that [women] are the carriers of the babies. Therefore, the ball is primarily in the mother's court to make the right choice. The father can definitely have an impact, but mother has the final say.

I would say the mother [is responsible for the fetus's wellbeing]. The mother should take care of the child. She should have the best interest of that child in mind. More than anyone, more than any program, more than any support, it falls to the woman. It's her baby. It's a part of her.

Because the woman is the gatekeeper, FASD is understood to be the direct result of her decision to "allow" alcohol to

\footnotetext{
${ }^{1}$ All participant interview excerpts have been edited to remove any personally identifying information and improve readability.
} 
enter her body. This way of thinking helps explain why participants held pregnant women narrowly and exclusively responsible for FASD.

Ultimately, it's the mother's fault for drinking if [FASD] is what happens. It's not really the child's fault. It's just too bad that the child has to suffer with that for the rest of his or her life, you know?

Everybody wants to blame somebody else for everything. And I think [women should have] accountability for what they do.

\subsubsection{Woman as Vessel}

Our analysis also supported previous research that has shown that when thinking about pregnancy, and especially FASD, people implicitly compare women to vessels (see, for example, Benoit et al., 2015; Rabuzzi, 1994). In this way, participants in this research saw pregnancy as a fundamental change in a woman's identity. Before pregnancy, a woman is seen as an independent agent with her own desires, goals, challenges, and responsibilities; she is responsible for the consequences of her decisions and actions. But upon pregnancy, her identity changes from an independent person to the carrier of another agent. Once the shift from self-interested agent to other-interested carrier-from individual to vessel-happens, the woman's life is understood as no longer her own. Instead of living for herself, the woman lives — or should live — in the service of the fetus's healthy development.

[Women] have to think about the wellbeing of their child. And they have to put that first, before their own wellbeing.

It would be nice if everybody thought about living healthy and doing all the right choices, but a woman that is pregnant I feel is obligated to, or should ideally, think beyond herself and to put as primary what's needed for her child.

\subsubsection{The Rational Actor}

We also found an assumption, underlying participants' thinking about alcohol use and FASD, in which behavior is the result of rational decisions. In other words, individuals carefully weigh the costs and benefits of a behavior before deciding to act. In this way, behaviors are understood to be the result of a conscious and rational decision calculus.

I get angry when I hear about FASD, because I just think, this is self-inflicted. Someone did this to this child.

I know my cousin has [FASD], because his mom wasn't very smart and made bad choices when she was pregnant with him.

Obviously, you have sex, with some luck [pregnancy is] gonna happen. And I don't know why they keep doing it. Because I know a lot of people, they had a lot of kids, but if you can't afford them or take care of them properly, then why are you doing it?

Participants understood alcohol consumption during pregnancy to be a deliberate choice; therefore, women who consume alcohol have consciously put their own wants ahead of the needs of the fetus. They are then seen as selfish and fundamentally immoral actors. This model fuels the stigma that attaches to women who drink during pregnancy, as they are seen to be making conscious but selfish decisions. The vitriol with which participants made this moral judgment was notable.

Selfish! How about selfish? No matter what your backstory is, you shouldn't be drinking when you are having a baby.

Participant: I think for the most part [drinking during pregnancy] would be out of selfishness.

Researcher: How so?

Participant: I think that she'd only be looking to take care of her own needs - not even needs, her wants. Her own wants. She wants to party, she wants to drink, and she's not thinking about what her baby needs.

Along with these foundational cultural models, we found a set of more specific patterns of reasoning that people use to think about FASD.

\subsection{Cultural Models of the Science of FASD}

When thinking about FASD specifically, participants drew on their understandings of biology and human development to explain how alcohol affects a fetus.

\subsubsection{Direct Chemical Transfer}

Participants seemed to understand the interaction between a mother and a fetus most clearly as a direct transmission of physical substances. Chemicals ingested by the mother (e.g., from food, alcohol, other drugs) were understood to be 
absorbed directly by the fetus and to affect it in various ways. Thus, in participant's thinking, if the mother consumes alcohol, then the fetus consumes alcohol.

Whatever you consume, the baby is going to consume it one way or the other. So, I think that until you've given birth [you should not drink alcohol], and then you can do whatever you want.

If you eat a lot of unhealthy foods, the baby's going to get that too. So, you want the baby to have nutritious foods.

Another assumption that was evident in our analysis is the idea that an amount of alcohol that is normal, or even inconsequential, for an adult woman is far more significant for a fetus because of its size. If a woman consumes any amount of alcohol, which is then passed along to the fetus, that quantity of alcohol is understood to be overwhelming given the fetus' small size. Therefore, even modest or minimal alcohol consumption is assumed to have devastating fetal effects.

I don't drink. So, if I had one drink, I'd probably get drunk off it. So, imagine something that's the size of your hand getting even a thimbleful. That's going to get them drunk.

\subsubsection{Critical Moment}

Participants shared the implicit understanding that a fetus is "unformed" and developing at a rapid pace. Fetal development is seen, therefore, as a critical time in which foundational aspects of brain and body are formed. According to this understanding, because the fetus is undergoing an intense process of development, it is particularly sensitive to alcohol exposure received from the woman who is drinking. Introducing alcohol at this stage is seen to dramatically derail development — especially brain development—creating severe and long-lasting consequences.

I think there's just so much development to a baby in such a small amount of time that any amount of alcohol [is harmful]. That's just going to, I think, stop whatever [development] is going on.

Researcher: Are there other effects of FASD? How long do the effects last?

Participant: I would assume it would be lifelong, because it's through your development-your creation, your conception. I would think it would be in every aspect of you. I think it would affect you lifelong, because it's part of you. It would be part of who you are. Maybe you're even born addicted, or [with] a taste for it. Because it's part of your blood and tissues and being.

Participants appeared aware that different brain and biological structures are under construction" at different points of the developmental process. When participants discussed FASD, they reasoned that the timing of prenatal alcohol exposure matters because it determines which neural and physiological structures will be interrupted and, therefore, what the effects of the alcohol exposure will be.

When you are drinking, you don't know what is developing, you don't know what's happening in there. So, what if it's that day the brain is developing? There could be some functional problems. There could be some learning disabilities or mental problems. I don't know. Just don't do it. Don't take the risk. But I would assume it affects the whole body from head to toe.

I know what it feels like if I drink alcohol. It does something in my head and things are different there. So if it's doing something to somebody that's not formed yet, if it changes anything in there, it probably can't change back. My head is already done forming. It already formed as a proper head should — as we like to say, "normal." So whatever change I do to it, unless I go completely drastic, it's going to be temporary. But I think if something is not finished growing, it would be permanent.

\subsubsection{Forever Damaged Minds}

Participants shared a basic definitional understanding of FASD; they understood FASD as a disorder associated with cognitive damage - fundamental changes to the brain that create learning and behavioral problems such as learning disabilities, impulsiveness, and aggression.

Stunted growth, stunted learning. Just generally, the kid doesn't have a chance.

The baby is born sometimes with physical abnormalities-you can tell by the distance of their eyes, for example. But it can't be specifically diagnosed as of yet, because cognitively you won't be able to tell if the child is FASD until school age, whether or not they've been affected by the alcohol. So, sometimes it's because they're not meeting their milestones. And other times they've met their milestones, but you start to see cognitively that they're not where they should be in school.

Participants shared an understanding that the cognitive damages that prenatal alcohol exposure causes are deep, foundational, and set in stone, and that effects are permanent. They understood that this damage creates lifelong 
problems with learning and behavior that lead to unemployment and criminal activity.

They're not able to function correctly. They'll learn at a different rate. Their mind capacity is much different from everybody else's. They could maybe have the tendency to make more bad decisions in life.

When they're kids, they would have difficulties in school and their home life. When they're adults, they could lead a life of crime. They could get into trouble more easily. Crime would be a big one. Not being able to cope with the syndrome they have would be another problem.

Because participants assumed that the minds of those with FASD are impaired, and that this impairment is permanent, they saw individuals with FASD as fundamentally different and apart from those they considered "normal."

I look at [people with FASD] as a jigsaw puzzle that doesn't quite fit in with the rest of the board. Something is wrong, that puzzle piece is broken. Because of that, they don't fit in, and because of that it's always a disruption to the rest of the puzzle pieces that go in there.

I guess it's just kind of more when you look in their eyes, like you can just see that stuff is not connecting. Concepts are not connecting. They're just in a different place.

People are going to look at [someone with FASD] and go, "Holy shit. I don't want my kid around your kid. I don't want to associate with you."

When participants used the Forever Damaged Minds model, they understood the life trajectory of someone with FASD to be as follows: (1) mother drinks, (2) child is born with FASD, (3) child has a cognitive disability and gets in trouble in school, and (4) child becomes a dysfunctional adult. They believe once a brain is damaged in this way, there is no repairing it, and little can be done to improve outcomes for the individual affected.

FASD was thus seen as permanent and largely immune to intervention. Participants explained that social services could help manage some of the effects of FASD on a person's life, but they did not think that these services could significantly improve outcomes.

3.2.4 Fate

Despite the ways in which the models described in Section 3 have led participants to assume that prenatal alcohol exposure has serious and long-term effects, they simultaneously acknowledged that in some cases, women consume alcohol and the fetus is not harmed. Even though participants acknowledged such circumstances, they were largely unable to explain how this could happen and defaulted to "fate" - the idea that sometimes biology just works in mysterious ways and is beyond human control and understanding.

I don't know [how FASD happens]. That gets into the finer things of biology, I think. In some cases, you just-you know what? I'm a big believer in fate. Unfortunately, some people are destined for it. And it's not fun. It's not fair, but as to why one kid has [FASD] and one kid doesn't, I don't know.

I know studies have proven that [alcohol is] harmful to a child, at least in excess it is. But other than that, I don't know too much of the scientific stuff, or how much a woman has to have. I know that zero tolerance is what's recommended. But I also know some that have had the odd glass of wine here or there, and everything's been fine.

Cultural Models of the Social Causes of FASD

Participants also had a set of models that they used to reason about the social causes of alcohol use and FASD.

\subsubsection{Escapism}

There was a shared assumption that alcohol use is a means of escaping difficult situations and unpleasant thoughts and feelings, including stressful life circumstances, mental health issues, intimate partner violence, and the frustration that results from a chronic lack of opportunity (e.g., education, jobs, services). In this way, alcohol was understood as a coping mechanism - a substance that is consumed to blunt negative emotions.

People drink [alcohol] for pleasure, and some people drink it because they're masking mental issues. If there's depression or anxiety, I feel like it gives them a temporary feeling of feeling better.

I figure that these women are in such shitty situations and having such shitty lives and maybe that lady doesn't want to have kids, but she is not capable of swallowing a pill every day or something. So she is going to have sex with her old man and she is going to get pregnant again, and she is probably just going to be like "fuck me" and just tune out as much as possible. And what's a fairly easy way of tuning out? This would be, check day comes around, you go get some liquor and just keep drinking.

While participants reasoned that the need to mentally escape a difficult situation is an explanation for drinking while 
pregnant, it is also clear, as seen in the second quote above, that the decision to escape ultimately results from a woman's lack of personal responsibility and control.

\subsubsection{Normalization}

Participants also explained alcohol use through an understanding that some people drink because "it's all they know"; that in certain communities, drinking is a cultural norm and has come to be expected behavior.

In discussing the causes of alcohol use, participants frequently cited cycles of dysfunction. They explained that if a person grew up around family or community members who drank excessively, the person will see this behavior as normal and will likely follow the same patterns as an adult. Participants explained that assumptions about what is normal and acceptable stay with people throughout their lives and influence decision-making. This way of thinking was often used to explain why a woman would use alcohol during pregnancy.

The cycle just keeps repeating itself. When you grow up in an alcoholic household or a stressed-out household that's poorly functioning, chances are you are going to have that kind of household yourself. And chances are your kids will. I've seen that.

If you are a little kid growing up watching this guy slaying drugs and act[ing] like a gang member and shoot[ing] at people, what are you going to do? Nine times out of 10 you are going to grow up to be exactly what you watched. That's what I mean by "circle."

\subsubsection{Lack of Awareness}

As noted above, participants were generally aware of FASD and the effects of alcohol on fetal development. They also assumed that this awareness was shared by others and that the dangers of consuming alcohol during pregnancy are well-known. That said, participants understood that a small proportion of cases of FASD result from women not knowing the risks of alcohol use during pregnancy. The assumption that follows is that if these women were made aware of the risks of alcohol use during pregnancy, they would immediately and easily choose not to drink.

I think you need to have the education, not just because of pregnancy, because of the harmful effects [of drinking]. You don't know what effect it's going to have.

While lack of awareness was understood to be a cause of alcohol consumption during pregnancy, participants believed that lack of awareness does not release women from taking personal responsibility for their decisions or excuse their failure to exercise willpower.

There's enough education here in Manitoba. You're being taught this in high school, even when I was in high school. So, if you're going to school, even if you go to those clinics to get medical help or whatever, all the pamphlets are there. There's some big signs. There's prenatal courses that the government offers. It's not like we keep it all a secret and you fend for yourself. No. It's all free information.

The Escapism, Normalization, and Lack of Awareness models were particularly prominent when participants talked about poverty and Indigenous communities. In thinking that alcohol use and FASD are more common in poor communities, participants reasoned that people living in poverty or in Indigenous communities regularly face stressful situations and lack opportunity, and therefore drink to escape these experiences (Escapism). Participants also reasoned that growing up in poverty or as an Indigenous person exposes people to the use of alcohol in a way that normalizes drinking (the Normalization model). Finally, participants assumed that the individuals who lacked an understanding of the negative developmental consequences of alcohol consumption during pregnancy were likely be Indigenous or living in poverty.

Researcher: Would you say that there are communities that are affected more by FASD than others? Or communities that are affected less than others?

Participant: Yes, I would say some communities are affected more.

Researcher: And why is that?

Participant: I think it all has to do with socioeconomic status. [...]

Researcher: Okay. So, why is it that socioeconomic status would be a factor in FASD?

Participant: Because I think that poverty in a lot of cases leads to higher rates of alcoholism and substance abuse.

Researcher: And why do you think that is the case?

Participant: A lack of education. I think that it's, again, what you've seen while growing up, kind of copycatting what you've seen. 


\subsubsection{Addiction Equals No Choice}

Participants understood addiction as the inability to control substance use and evoked addiction as an explanation of why some women drink during pregnancy.

I'm assuming [a woman who is pregnant] is drinking because she's addicted. The only thing I can think of is she is drinking because she is addicted. I assume everybody knows the effects of drinking while you are pregnant so why would you do it? The only reason I could possibly think is it's out of your control.

Why would a pregnant woman continue to drink? Maybe because she might have an alcohol addiction. She might like it so much that she just can't control herself. She can't restrain herself from having it, because it's really difficult to get off of things that you're addicted to. You have to take it down a notch. But even then, if you do, you just can't quit doing it right at once.

Addiction was understood as a disease that robs individuals of control over their actions and behaviors. It is important to note that this model is in tension with Individualism and Rational Actor models of behavior discussed above, in that addiction is understood as a phenomenon in which rational decision-making and individual control are suspended. However, the Addiction Equals No Choice model seemed less powerful in shaping thinking than the more dominant individualistic models. In other words, while participants reasoned that addiction explains alcohol use during pregnancy among a small minority of women, they tended to default to ways of thinking that enabled them to hold an individual woman responsible.

\section{Solutions}

When asked to think about what could be done to address FASD, participants conveyed a strong sense of fatalism. This fatalism was most clearly seen in the lack of engagement in talking about solutions, a lack of conviction that meaningful changes could be made, and explicit opinions that preventing or remediating FASD was not likely to happen.

Analysis showed that this fatalism stems in part from the way that people see women and their decisions as exclusively responsible for FASD, and the degree to which they see selfishness and lack of willpower as difficult, if not impossible, to prevent. This fatalism also appears to stem from participants' thinking about how women might not be solely responsible for FASD. If people believe that alcohol use is either something that has been deeply embedded into communities over time or beyond individual control due to the disease of addiction, then use is impervious to remediation. Finally, and most powerfully, when participants focused on the belief that damage is permanent, they showed little motivation to engage with potential solutions and had little conviction in them. In short, participants' most dominant ways of thinking about FASD all led to a common conclusion: there is ultimately nothing that can be done to prevent FASD or improve outcomes for those with the disorder.

I don't know. They keep saying, educate people, but I think if it's not in them, you can educate all you want, but I don't know if it's gonna make a difference.

I don't think any regulations around alcohol would make a difference. If a person wants to drink and they are 12 years old, they are going to drink. If they want to drink and they are broke and they are 40, they are going to find a way to drink. If a person is predisposed and wants to make those choices, they are going to make them. They are going to find a way to make the alcohol, or steal it, buy it, prostitute for it.

In response to more questions and further probing about actions that could be taken to address FASD, participants were able to generate a set of ideas that they thought could be taken to reduce the number of cases of FASD in Manitoba. Each of these ideas appeared to be informed by one or more of the cultural models discussed above.

Solution No. 1: Encourage pregnant women to exercise willpower and abstain from alcohol.

When engaged in discussions that seemed to be shaped by Individualism, participants frequently suggested that because FASD is the result of a woman's choices, one way to address the issue is to encourage women to be more disciplined in their decision-making when it comes to consuming alcohol during pregnancy.

Solution No. 2: Educate women about the dangers of consuming alcohol while pregnant.

Participants focused on how providing information to women might be one way of decreasing rates of FASD. According to this thinking, some cases, albeit few, of FASD result from women simply not knowing about the harmful effects of consuming alcohol during pregnancy. If women knew how harmful consuming alcohol during pregnancy could be, fewer would choose to drink.

Participants acknowledged that there are currently many awareness campaigns about FASD in Manitoba. While they could see the potential of education, they also thought that information is already easily accessible, and therefore were skeptical about whether such efforts could ultimately make any difference. 
Solution No. 3: Shame women who drink while pregnant.

Participants saw the stigmatization of pregnant women who drink as an important strategy to use to address, and potentially, prevent FASD. Participants reasoned (drawing on the idea of a Rational Actor) that, if women know that they will be stigmatized if they choose to drink, they will weigh the costs of this choice differently and decide that the benefits do not warrant the costs. As a result, more women will decide not to drink while pregnant.

Solution No. 4: Refuse to serve women alcohol.

Participants reasoned that another solution to FASD would be giving those who serve alcohol the power and legal prerogative to refuse service to women who are pregnant. This solution seems to follow from the metaphor of Woman as Vessel. If the fetus is the priority and more important than the woman who is pregnant, it seems appropriate to limit the woman's choices and rights.

Solution No. 5: Provide support to pregnant women.

Finally, participants occasionally nodded toward the importance of supporting pregnant women. Support was seen to potentially come from the family, the health care system, or social services. Though the specifics of these examples were vague, the overall goal of their provision was to help women make better choices.

This solution appeared to stem from participants' acknowledgement that there are factors that influence women's choices. This is the solution that most closely approximates the preventative approaches advocated by experts (Fond et al., 2017). However, it should be noted that this was not the easiest solution for participants to generate and explain during interviews, and their thinking was easily overtaken by fatalism about the effectiveness of social services.

\section{Discussion}

These cultural models have a number of implications for communicators as well as those studying the connection between communication, public thinking, and public health policy. Below we identify several of the most significant implications of this work and conclude with communication recommendations that our analysis suggests.

The foundational cultural models of pregnancy-Individualism, Gatekeepers, Woman as Vessel, and Rational Actor - make it difficult for people to consider the social determinants of FASD. Together, the four foundational cultural models of responsibility and decision-making described above shape a belief that women who are pregnant are the sole entities responsible for fetal wellbeing and therefore FASD. These patterns of reasoning make it difficult for people to think about the role of the social environment in shaping fetal development. People's difficulty in considering social determinants is a key challenge for those communicating about a public health approach to preventing and addressing FASD

Understanding the causes of FASD exclusively in terms of individual choice limits the range of solutions that people can see as appropriate and effective. If people understand FASD as the result of poor decision-making, then the solutions that appear appropriate are those that influence decisions and choice. These solutions include educational interventions and punitive actions that people think discourage negative choices and shape behavior. These ways of thinking make it difficult for people to understand, appreciate, and support many of the solutions advocated by experts - especially those that focus on social determinants.

The foundational models of women and pregnancy and the Forever Damaged Minds model help explain the stigmatization that many women report encountering. Reasoning with the foundational cultural models described above, FASD is seen quite simply as the result of decisions made by a weak or selfish person. This perspective fuels stigma and reinforces difficulties in addressing this issue. If FASD is the result of weak or immoral individuals unable or unwilling to make the right choice, it becomes easy to focus blame on individuals, hard to see the role of context, and harder still to support systemic solutions. Seeing those with FASD as fundamentally impaired "others" feeds the stigma associated with this issue. The strong stigma attached to FASD, which people believe could (and should) be leveraged to motivate women to stop drinking, makes it even more difficult to talk openly about what can be done. Understanding people with FASD as having Forever Damaged Minds also prevents people from thinking deeply about how wraparound social services could help people with FASD lead more fulfilling lives. For these reasons, communicating about the prevention of FASD and the provision of supportive services for those with FASD is challenging.

The Forever Damaged Minds and Fate cultural models create a strong sense of fatalism around FASD issues. Because FASD is understood in terms of permanent dysfunction, it is exceedingly difficult to get the public to engage in solutions thinking or to generate support for programs and policies designed to support those with FASD. The public has limited ways to think about how the life of a person with FASD could be improved; therefore, an important task for communicators is to be explicit and explanatory in their discussion of solutions.

However, there are elements of several of the cultural models identified that align more closely with the perspectives of 
FASD researchers, medical professionals, and advocates. For example, people's thinking about alcohol use as an escape is a rare way of thinking that leads people to recognize how context and experiences can influence behavior. When thinking with the Escapism model, people are more able to consider the broader context in which actions are taken, and they do not hold women completely responsible. The Addiction Equals No Choice model is another potentially productive pattern of thinking. This model, which helps people think about why a woman would not be able to stop drinking if she is pregnant, challenges people's assumption that individuals are solely responsible for making rational choices. Communicators can build on this model to show how supports - rather than individual willpower-are needed to prevent and address this issue.

Our research suggests a set of strategies that can be used to engage people in more productive discussions about FASD. More effective communication about this issue is important; without a more open, productive dialogue, the large-scale political and practical, let alone cultural, change necessary to address and prevent FASD will not occur. While more work must be done to design and test specific communication strategies that can best overcome the challenges outlined above, the following recommendations appear promising.

1. Reframe FASD as a contextual, not individual, issue. Most definitions of FASD emphasize that FASD includes a spectrum of possible effects that can vary dramatically depending on the person. However, the cause of FASD is typically described simply as "a woman drank alcohol while pregnant," a fact with which people seem generally familiar. Communicators should put forward a definition of FASD - in educational materials, in schools, in doctors' offices, on websites - that describes FASD as a disorder caused by biological and social factors. While alcohol consumption is indeed the direct cause of FASD, a definition that focuses on consumption masks the important social factors that affect consumption and allows people to fill in the missing details with stigma and blame directed at women.

2. Tell stories that make context clear. To counter the tendency to explain FASD by blaming a woman's individual decisions, communicators should tell stories that frame FASD as a social issue with systemic causes and collective solutions. It is quite common for stories about FASD to narrowly focus on individuals' circumstances. While stories often depend on including individuals that the audience identifies with, stories must include exposition of the systems, programs, and social factors that facilitate positive outcomes (or, in contrast, that challenge positive outcomes). But stories that are narrowly and exclusively about individuals reinforce individualistic thinking and focus people's attention on character flaws" as the explanations for poor outcomes (Iyengar, 1996). This is particularly concerning for stories about FASD, because people's foundational cultural models of women and pregnancy already focus thinking on individual willpower and character-obscuring the relevant contextual factors. Instead, programs, social factors, and systems must also be characters in stories to help correct the public's tendency to focus on individual women and their individual choices to the exclusion of social determinants.

3. Counter fatalistic thinking with detailed descriptions of effective solutions. Helping people overcome their fatalism is one of the key challenges that emerges from this work. The task of helping the public see that improvement is possible is more urgent than the task of making the public see FASD as a problem; therefore, in addition to framing communications with values, communicators should reinforce the idea that positive change is possible. To do this, they must explain the details of how programs and policies lead to better outcomes. For example, communicators must articulate how programs and policies can help people with FASD lead full lives with specificity. The public needs explanations - not just lists or descriptions - of ways that supportive services and smart policies, including better diagnoses and funding for research, can be effective in improving outcomes for those affected by FASD.

We hope that this research highlights the potential of cultural models analysis as a powerful strategic communications research tool. Understanding how members of a population think about an issue reveals both communication challenges and potentially effective strategies. With a deeper understanding of the culture that communicators send their messages into, it is our belief that those messages can be more effectively framed, advancing public discourse on key social issues and driving public will as a precursor to significant policy change.

\section{References}

Bell, K., McNaughton, D., \& Salmon, A. (2009). Medicine, morality, and mothering: Public health discourses on fetal alcohol exposure, smoking around children, and childhood over nutrition. Critical Public Health, 19(2), 155-170. https://doi.org/10.1080/09581590802385664

Benford, R., \& Snow, D. (2000). Framing processes and social movements: An overview and assessment. Annual Review of Sociology, 26, 611-639. https://doi.org/10.1146/annurev.soc.26.1.611

Benoit, C., Magnus, S., Phillips, R., Marcellus, L., \& Charbonneau, S. (2015). Complicating the dominant morality discourse: Mothers' and fathers' constructions of substance use during pregnancy and early 
parenthood. International Journal for Equity in Health, 14(1), 72. https://doi.org/10.1186/s12939-015-0206-7

Burd, L. (2016). Fetal alcohol spectrum disorder: Complexity from comorbidity. The Lancet, 387. https://doi.org/10.1016/S0140-6736(15)01346-X

Chudley, A. E., Conry, J., Cook, J. L., Loock, C., Rosales, T., \& LeBlanc, N. (2005). Fetal alcohol spectrum disorder: Canadian guidelines for diagnosis. Canadian Medical Association Journal, 172(5). https://doi.org/10.1503/cmaj.1040302

Connolly-Ahern, C., \& Broadway, S. C. (2008). "To booze or not to booze?" Newspaper coverage of fetal alcohol spectrum disorders. Science Communication, 29(3), 362-385. https://doi.org/10.1177/1075547007313031

D'Andrade, R. (1987). A folk model of the mind. In D. Holland and N. Quinn (Eds.), Cultural models in language and thought (pp. 1121-1150). Cambridge: Cambridge University Press. https://doi.org/10.1017/CBO9780511607660.006

D’Andrade, R. (1995). Moral models in anthropology. Current Anthropology, 36(3), 399-408. https://doi.org/10.1086/204377

D'Andrade, R. (2005). Some methods for studying cultural and cognitive structures. In N. Quinn (Ed.), Finding culture in talk: A collection of methods (pp. 83-104). New York City: Palgrave Macmillan. https://doi.org/10.1007/978-1-137-05871-3_3

Eguiagaray, I., Scholz, B., \& Giorgi, C. (2016). Sympathy, shame, and few solutions: News media portrayals of fetal alcohol spectrum disorders. Midwifery, 40, 49-54. https://doi.org/10.1016/j.midw.2016.06.002

Fond, M., Kendall-Taylor, N., Volmert, A., Gertein Pineau, M., \& L'Hôte, E. (2017). Seeing the spectrum: Mapping the gaps between expert and public understandings of fetal alcohol spectrum disorder in Manitoba, 46-47. Washington, DC: Frame Works Institute.

Golden, J. L. (2000). "A Tempest in a Cocktail Glass": Mothers, alcohol, and television, 1977-1996. Journal of Health Politics, 25(3), 473-498. https://doi.org/10.1215/03616878-25-3-473

Hirschman, E. (2003). Men, dogs, guns, and cars: The semiotics of rugged individualism. Journal of Advertising, 32(1), 9-22. https://doi.org/10.1080/00913367.2003.10601001

Iyengar, S. (1996). Framing responsibility for political issues. The Annals of the American Academy of Political and Social Science, 546, 59-70. https://doi.org/10.1177/0002716296546001006

Kendall-Taylor, N. (2012). Conflicting models of mind: Mapping the gaps between expert and public understandings of child mental health. Science Communication, 34(6), 695-726. https://doi.org/10.1177/1075547011429200

Kendall-Taylor, N., McCollum, C., \& Manuel, T. (2009). Caught between osmosis and environments: Mapping the gap between the expert and the public understandings of the role of executive function. Washington, DC: Frame Works Institute.

Lindland, E., Kendall-Taylor, N., Haydon, A., \& Fond, M. (2016). Gauging aging: Expert and public understanding of aging in America. Communication and the Public, 1(2), 211-229. https://doi.org/10.1177/2057047315625340

Lowe, P., Lee, E., \& Yardley, L. (2010). Under the influence? The construction of fetal alcohol syndrome in UK newspapers. Sociological Research Online, 15(4). https://doi.org/10.5153/sro.2225

Lupton, C., Burd, L., \& Harwood, R. (2004). Cost of fetal alcohol spectrum disorders. American Journal of Medical Genetics Part C: Seminars in Medical Genetics, 127(1), 42-50. https://doi.org/10.1002/ajmg.c.30015

Millichap, J. G., \& Millichap, J. J. (2014). Fetal alcohol spectrum disorder prevalence. Pediatric Neurology Briefs, 28(12), 94-95. https://doi.org/10.15844/pedneurbriefs-28-12-8

Pei, J., Leung, W., Jampolsky, F., \& Alsbury, B. (2016). Experiences in the Canadian criminal justice system for individuals with fetal alcohol spectrum disorders: Double jeopardy? Canadian Journal of Criminology and Criminal Justice, 58(1), 56-86. https://doi.org/10.3138/cjccj.2014.E25

Popova, S., Lange, S., Burd, L., Chudley A. E., Clarren, S. K., \& Rehm, J. (2013). Cost of fetal alcohol spectrum disorder diagnosis in Canada. PLoS ONE, 8(4), e60434. https://doi.org/10.1371/journal.pone.0060434

Popova, S., Stade, B., Johnston, M., MacKay, H., Lange, S., Bekmuradov, D., \& Rehm, J. (2011). Evaluating the cost of fetal alcohol spectrum disorder. Journal of Studies on Alcohol and Drugs, 72(1), 163-164. https://doi.org/10.15288/jsad.2011.72.163

Quinn, N. (2005a). Finding culture in talk: A collection of methods. New York City: Palgrave Macmillan. 
https://doi.org/10.1007/978-1-137-05871-3

Quinn, N. (2005b). How to reconstruct schemas people share, from what they say. In N. Quinn (Ed.), Finding culture in talk: A collection of methods (pp. 35-82). New York City: Palgrave Macmillan. https://doi.org/10.1007/978-1-137-05871-3_2

Quinn, N., \& Holland, D. (1987). Culture and cognition. In D. Holland and N. Quinn (Eds.) Cultural models in language and thought (pp. 3-42). Cambridge UK: Cambridge University Press. https://doi.org/10.1017/CBO9780511607660.002

Rabuzzi, K. A. (1994). Mother with child: Transformations through childbirth. Bloomington: Indiana University Press.

Riley, E. P., Infante, M. A., \& Warren, K. R. (2011). Fetal alcohol spectrum disorders: An overview. Neuropsychological Review, 21(2), 73. https://doi.org/10.1007/s11065-011-9166-x

Roozen, S., Peters, G., Kok, G., Townend, D., Nijhuis, J., \& Curfs, L. (2016). Worldwide prevalence of fetal alcohol spectrum disorders: A systematic literature review including meta-analysis. Alcoholism: Clinical and Experimental Research, 40(1). https://doi.org/10.1111/acer.12939

Ryan, D. M., \& Bonnett, D. M., \& Gass, C. B. (2006). Sobering thoughts: Town hall meetings on fetal alcohol spectrum disorder. American Journal of Public Health, 92(12), 2098-2101. https://doi.org/10.2105/AJPH.2005.062729

Shore, B. (1996). Culture in mind: Cognition, culture, and the problem of meaning. New York City: Oxford University Press.

Stade, B., Ali, A., Bennett, D., Campbell, D., Johnston, M., Lens, C., Tran, S., \& Koren, G. (2009). The burden of prenatal exposure to alcohol: Revised measurement of cost. Canadian Journal of Clinical Pharmacology, 16(1), e91-e102.

Stewart, M., \& Glowatski, K. (2014). Frontline police perceptions of fetal alcohol spectrum disorder in a Canadian province. Police Journal: Theory, Practice and Principals, 87(1), 17-27. https://doi.org/10.1350/pojo.2014.87.1.648

Strauss, A. L., \& Corbin, J. (2015). Basics of qualitative research: Techniques and procedures for developing grounded theory. Los Angeles, CA: Sage Publications.

Strauss, C. (2005). Analyzing discourse for cultural complexity. In N. Quinn (Ed.), Finding culture in talk: A collection of methods (pp. 203-242). New York City: Palgrave Macmillan. https://doi.org/10.1007/978-1-137-05871-3_6

Triandis, H. (1989). The self and social behavior in differing cultural contexts. Psychological Review, 96, 506-520. https://doi.org/10.1037/0033-295X.96.3.506

\section{Copyrights}

Copyright for this article is retained by the author(s), with first publication rights granted to the journal.

This is an open-access article distributed under the terms and conditions of the Creative Commons Attribution license which permits unrestricted use, distribution, and reproduction in any medium, provided the original work is properly cited. 